\title{
HidroEsta, software para cálculos hidrológicos y estadísticos aplicados a la Hidrología
}

\author{
Máximo Villón Béjar. \\ mvillon@itcr.ac.cr, \\ Instituto Tecnológico de Costa Rica
}

\section{Resumen.}

Este trabajo de investigación desarrollado en el 2004 en el TEC, se orientó a la elaboración de una herramienta computacional bajo el título HidroEsta, software para cálculos hidrológicos, utilizando Visual Basic. El cual pretende ser una aplicación que permita facilitar y simplificar los cálculos laboriosos, que se deben realizar en los estudios hidrológicos.

El software permite el cálculo de los parámetros estadísticos, cálculos de regresión lineal, no lineal, simple y múltiple así como regresión polinomial, evaluar si una serie de datos se ajustan a una serie de distribuciones, calcular a partir de la curva de variación estacional o la curva de duración, eventos de diseño con determinada probabilidad de ocurrencia, realizar el análisis de una tormenta y calcular intensidades máximas, a partir de datos de pluviogramas, los cálculos de aforos realizados con molinetes o correntómetros, el cálculo de caudales máximos, con métodos empíricos y estadísticos, cálculos de la evapotranspiración y cálculo del balance hídrico.

En la investigación se probaron diferentes métodos numéricos para la solución de las ecuaciones, seleccionándose el más adecuado para cada situación.

El producto del trabajo proporciona al ingeniero civil, agrícola, agrónomo, hidrólogo y otros especialistas que trabajen en este campo, una herramienta que permite realizar cálculos, simulaciones rápidas, y determinar los caudales o precipitaciones de diseño.

Palabras clave:

Estadística, Probabilidades, Funciones de distribución, Hidrología, Caudales, Precipitaciones, Momentos lineales.

\begin{abstract}
This investigation work was guided to the elaboration of a computation system named HidroEsta, software for hydrological calculations, using Visual Basic. Which seeks to be an application that allows to facilitate and to simplify the laborious calculations that should be carried out in the hydrological studies.

The software allows the calculation of the statistical parameters, calculations of lineal regression, not lineal, simple and multiple as well as regression polinomial, to evaluate if a series of data is adjusted to a series of distributions, to calculate starting from the curve of seasonal variation or the duration curve, design events with certain occurrence probability, to carry out the analysis of a storm and to calculate maximum intensities, the calculations of seating capacity carried out with brandishes, the calculation of maximum flows, with empiric and statistical methods, calculations of the evapotranspiration and calculation of the water balance.
\end{abstract}


In the investigation different numeric methods were proven for the solution of the equations, being selected the most appropriate for each situation.

The product of the work provides the civil, agricultural, agricultural engineer, hidrologic engineer and other specialists that work in this field, a tool that allows to carry out calculations, quick simulations, and to determine the flows or design precipitations.

Keywords: Statistics, Probabilities, Distribution functions, Hydrology, Flows, Precipitations, L-moments.

\subsection{Introducción}

Los estudios hidrológicos requieren del análisis de cuantiosa información hidrometeorológica; esta información puede consistir de datos de precipitación, caudales, temperatura, evaporación, etc.

Los datos recopilados, solo representan una información en bruto, pero si éstos se organizan y analizan en forma adecuada, proporcionan al hidrólogo una herramienta de gran utilidad, que le permite tomar decisiones en el diseño de estructuras hidráulicas.

Para realizar los cálculos, los hidrólogos tienen que enfrentarse a una serie de problemas, debido a que:

- El procesamiento de la información que se tienen que realizar son bastante laboriosos.

- Las ecuaciones que se tienen que solucionar, en la mayoría de los casos son muy complejas, y para su solución se requiere del uso de métodos numéricos.

- Las simulaciones que se realizan manualmente consumen mucho tiempo, debido a los cálculos que se requieren.

Por lo laborioso del proceso de la información y de los cálculos se puede incurrir en errores, por lo que se requiere de un software que brinde al hidrólogo de una herramienta que le permita simplificar todos estos procesos, e inclusive permitirle simular sus resultados, permitiendo con esto optimizar su diseño.

HidroEsta, es una herramienta que facilita y simplifica los cálculos laboriosos, y el proceso del análisis de la abundante información que se deben realizar en los estudios hidrológicos. Puede adquirirse con el autor llamando al número 8837-6413. Por la gran aceptación que ha tenido este trabajo a nivel mundial y con base a las sugerencias de los usuarios, en estos momentos se está trabajando con Hidroesta 2, la misma que estará disponible a finales de julio del 2012.

\subsection{Importancia}

HidroEsta representa una contribución para simplificar los estudios hidrológicos, es importante porque:

- Proporciona una herramienta novedosa y fácil de utilizar para el ingeniero civil, ingeniero agrícola, ingeniero agrónomo y otros especialistas que trabajen en el campo de los estudios hidrológicos.

- Permite simplificar el proceso de la abundante información y los cálculos laboriosos.

HidroEsta, software para cálculos hidrológicos. Máximo Villón B.

Derechos Reservados (C 2012 Revista digital Matemática, Educación e Internet (www.tec-digital.itcr.ac.cr/revistamatematica/) 
- Permite a partir de la información proporcionada, simular los parámetros de diseño de las estructuras a construir.

- Reduce enormemente el tiempo de cálculo.

- Permite obtener un diseño óptimo y económico.

\subsection{Descripción del sistema}

El sistema permite resolver los problemas más frecuentes que se presentan en los cálculos hidrológicos, los cuales son:

- El cálculo de los parámetros estadísticos, para datos agrupados y no agrupados, tanto con los momentos tradicionales como con momentos lineales.

- Cálculos de regresión lineal, no lineal, simple y múltiple así como regresión polinomial.

- Evaluar si una serie de datos se ajustan a una serie de distribuciones: normal, log-normal de 2 y 3 parámetros, gamma de 2 y 3 parámetros, log-Pearson tipo III, Gumbel y log-Gumbel, tanto con momentos ordinarios, como con momentos lineales. Si la serie de datos se ajusta a una distribución, permite calcular por ejemplo caudales o precipitaciones de diseño, con un período de retorno dado o con una determinada probabilidad de ocurrencia.

- Calcular a partir de la curva de variación estacional o la curva de duración, eventos de diseño con determinada probabilidad de ocurrencia.

- Realizar el análisis de una tormenta y calcular intensidades máximas, a partir de datos de pluviogramas, así como la intensidad máxima de diseño para una duración y periodo de retorno dado, a partir del registro de intensidades máximas. También permite el cálculo de la precipitación promedio por los métodos promedio aritmético, polígono de Thiessen e isoyetas.

- Los cálculos de aforos realizados con molinetes o correntómetros.

- El cálculo de caudales máximos, con métodos empíricos (racional y Mac Math) y estadísticos (Gumbel y Nash).

- Cálculos de la evapotranspiración con los métodos de Thorthwaite, Blaney-Criddle, Penman, Hargreaves y cálculo del balance hídrico.

La solución a estos problemas requiere de cálculos mediante el uso de métodos numéricos, y desarrollo de series. La aplicación, proporciona una ayuda para que el usuario pueda usar sin ninguna dificultad el software, y también donde se da explicación de los conceptos y ecuaciones utilizadas.

Con HidroEsta, es posible almacenar la información de entrada en archivos, a fin de repetir los cálculos las veces que se desee. Los datos procesados y resultados obtenidos, se almacenan en archivos de textos en formato .RTF, de donde se puede agregar a un documento .DOC cuando se quiera elaborar un informe. 


\subsection{Menú principal}

El sistema HidroEsta, tiene un Menú Principal, como el que se muestra en la Figura 1, el cual permite al usuario elegir la opción deseada según el cálculo que tenga que realizar.

HidroEsta, software para cálculos hidrológicos y estadísticos aplicados a la hidrología

Parámetros Estadísticos Regresión Distribuciones Curvas características Precipitación Aforo Caudales máximos Evapotranspiración Ayuda

Figura 1.1 Menú Principal de HidroEsta

Las opciones del menú principal son:

\begin{tabular}{|c|c|}
\hline Opciones & Descripción \\
\hline $\begin{array}{l}\text { Parámetros Estadísti- } \\
\text { cos }\end{array}$ & $\begin{array}{l}\text { Permite el cálculo de los parámetros estadísticos, para datos agrupa- } \\
\text { dos y no agrupados, tanto con los momentos tradicionales como con } \\
\text { momentos lineales (L-moments). }\end{array}$ \\
\hline Regresión & $\begin{array}{l}\text { Permite el cálculo de las ecuaciones de regresión lineal, no lineal, simple } \\
\text { y múltiple así como la regresión polinomial. }\end{array}$ \\
\hline Distribuciones & $\begin{array}{l}\text { Permite evaluar si una serie de datos se ajustan a una serie de dis- } \\
\text { tribuciones: normal, log-normal con } 2 \text { y } 3 \text { parámetros, gamma con } 2 \\
\text { y } 3 \text { parámetros, log-Pearson tipo III, Gumbel y log-Gumbel, tanto con } \\
\text { momentos ordinarios, como con momentos lineales. Si la serie de datos } \\
\text { se ajusta a una distribución, permite calcular por ejemplo caudales o } \\
\text { precipitaciones de diseño, con un período de retorno dado o con una } \\
\text { determinada probabilidad de ocurrencia. }\end{array}$ \\
\hline Curvas características & $\begin{array}{l}\text { Permite calcular a partir de la curva de variación estacional o la curva } \\
\text { de duración, eventos de diseño con determinada probabilidad de ocur- } \\
\text { rencia. }\end{array}$ \\
\hline Precipitación & $\begin{array}{l}\text { Permite realizar el análisis de una tormenta y calcular intensidades } \\
\text { máximas, a partir de datos de pluviogramas, así como la intensidad } \\
\text { máxima de diseño para una duración y periodo de retorno dado, a par- } \\
\text { tir del registro de intensidades máximas. También permite el cálculo de } \\
\text { la precipitación promedio por los métodos promedio aritmético, polí- } \\
\text { gono de Thiessen e isoyetas. }\end{array}$ \\
\hline Aforo & $\begin{array}{l}\text { Permite los cálculos de aforos realizados con molinetes o correntóme- } \\
\text { tros. }\end{array}$ \\
\hline Caudales máximos & $\begin{array}{l}\text { Permite el cálculo de caudales máximos, con métodos empíricos } \\
\text { (racional y Mac Math) y estadísticos (Gumbel y Nash). }\end{array}$ \\
\hline Evapotranspiración & $\begin{array}{l}\text { Permite cálculos de la evapotranspiración con los métodos de Thorth- } \\
\text { waite, Blaney-Criddle, Penman, Hargreaves y cálculo del balance hí- } \\
\text { drico. }\end{array}$ \\
\hline Ayuda & $\begin{array}{l}\text { Permite que el usuario pueda utilizar la aplicación y pueda consultar } \\
\text { las ecuaciones que se utilizan en los cálculos. }\end{array}$ \\
\hline
\end{tabular}




\subsection{Pantallas de trabajo}

Se presenta como ejemplo las pantallas para el:

- Ajuste de una serie de datos a una distribución normal

- Análisis de una tormenta

\subsubsection{Ajuste de una serie de datos a una distribución normal}

La pantalla para el cálculo del ajuste de una serie de datos a una distribución normal, se muestra en la figura 2. El usuario debe ingresar la serie de datos

En la opción del ajuste a la distribución normal, el programa:

- Calcula los parámetros estadísticos tanto con momentos ordinarios como con los momentos lineales.

- Realiza la prueba de bondad de ajuste con el método de Smirnov-Kolmogorov para ver si los datos de la serie se ajustan a la distribución teórica normal.

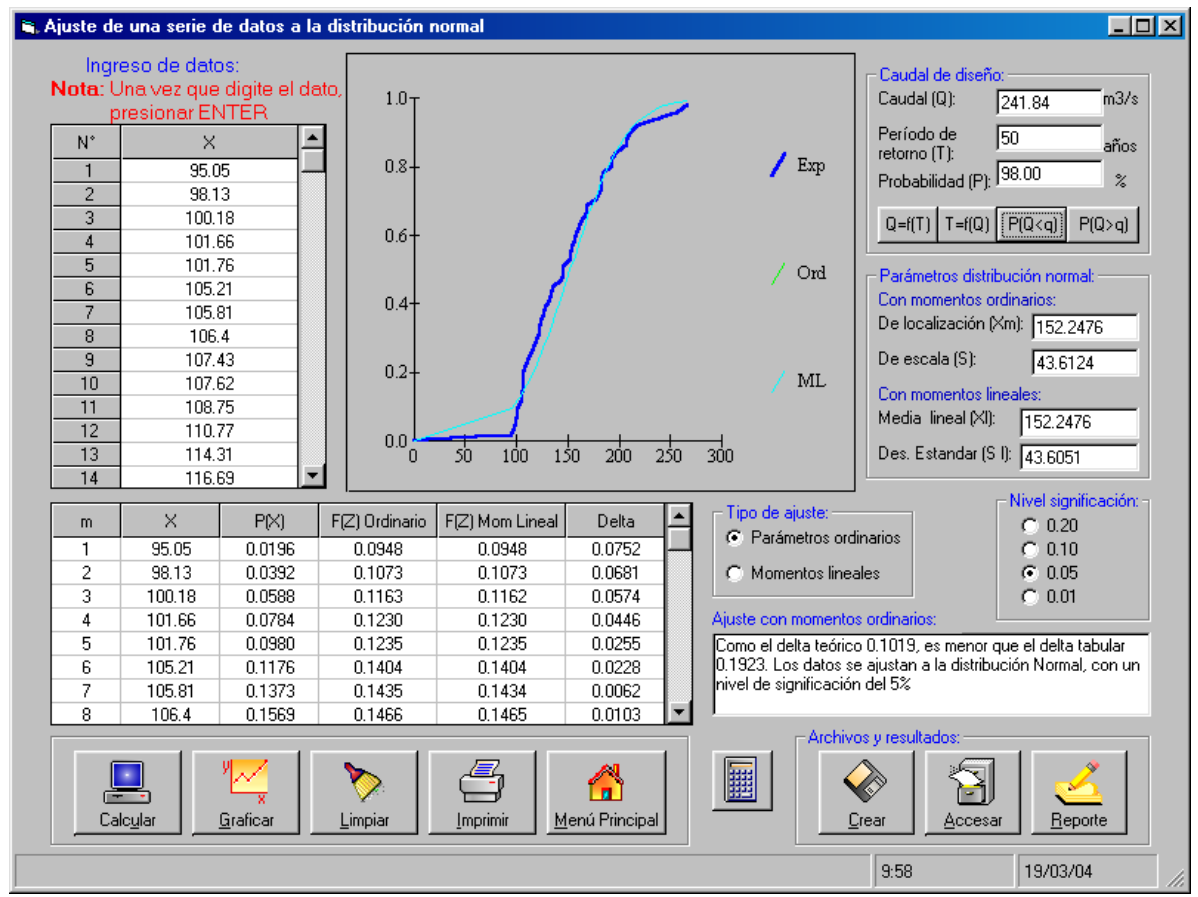

Figura 1.2 Pantalla para el cálculo de la distribución normal

- Si el ajuste es bueno, permite el cálculo del caudal de diseño para un período de retorno dado.

- Permite también el cálculo de la probabilidad de que un evento sea menor que éste, o que sea igualado o superado.

- Permite guardar la serie de datos ingresados.

- Permite generar un reporte en formato RTF, con los datos ingresados y los resultados obtenidos

HidroEsta, software para cálculos hidrológicos. Máximo Villón B.

Derechos Reservados (C) 2012 Revista digital Matemática, Educación e Internet (www.tec-digital.itcr.ac.cr/revistamatematica/) 


\subsubsection{Análisis de una tormenta}

La pantalla para el cálculo del análisis de una tormenta obtenida de un pluviograma, se muestra en la figura 3. El usuario debe ingresar los datos de los tiempos parciales y lluvias parciales, obtenidas de un pluviograma.

En la opción análisis de una tormenta, el programa:

- Calcula las intensidades para cada período

- Calcula el valor de la intensidad máxima e indica su duración

- Grafica el histograma o la curva masa

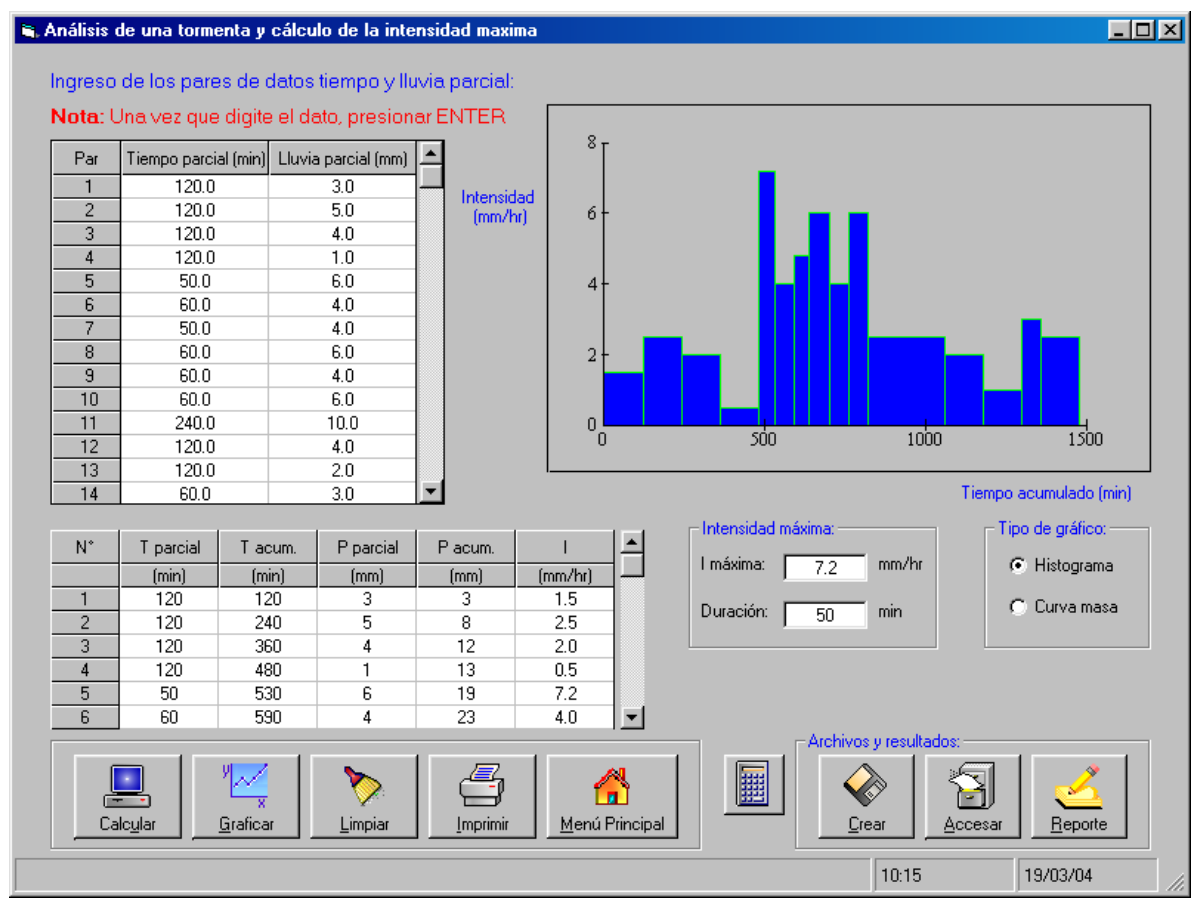

Figura 1.3 Análisis de un pluviograma.

\subsection{Impacto}

HidroEsta es una tecnología computacional que no existe en el mercado, por lo que llena un vacío que existía en los cálculos, a los que se dedican a los estudios hidrológicos, su uso se ha popularizado tanto en Costa Rica, como en Nicaragua, Guatemala, México, Perú, Chile, Cuba, Colombia, Ecuador, Bolivia, Argentina, Paraguay y Uruguay, a través de la divulgación que se ha realizado.

Hidroesta, se han presentado en los siguientes congresos:

- XI Encuentro Científico Internacional (ENCI2005), organizado por la Universidad de Ingeniería, del 2 al 5 de enero del 2005. Lima- Perú.

- VIII Congreso Internacional y del Caribe de Ingeniería Agrícola, organizado por la Universidad de Ingeniería Agrícola y la Asociación Latinoamericana y del Caribe de Ingeniería Agrícola, del 07 al 09 de mayo del 2008. Managua-Nicaragua.

HidroEsta, software para cálculos hidrológicos. Máximo Villón B.

Derechos Reservados (C) 2012 Revista digital Matemática, Educación e Internet (www.tec-digital.itcr.ac.cr/revistamatematica/) 
- VI Congreso Nacional de Estudiantes de Ingeniería Agrícola, organizado por la Escuela Profesional de Ingeniería Agrícola de la Universidad Santiago Antunez de Mayolo, del 12 al 16 de octubre del 2009. Huaraz - Perú.

- VI Congreso Internacional Ingeniería Agrícola (CIIACH), organizado por la Universidad de Concepción, del 11 al 13 de enero del 2010. Chillán - Chile.

- 21st Century Watershed Technology Conference 2010, organizado por la ASABE y la EARTH, del 21 al 24 de febrero del 2010. Guácimo - Costa Rica

- I Congreso Latinoamericano y del Caribe de Estudiantes de Ingeniería Agrícola, organizado por la Facultad de Ingeniería Agrícola de la Universidad Nacional Agraria la Molina, del 9 al 13 de agosto del 2010. Lima-Perú.

- XVIII Congreso Nacional de Estudiantes de Ingeniería Civil, organizado por la Facultad de Ingeniería Civil de la Universidad Privada Antenor Orrego, del 23 al 28 de agosto del 2010. Trujillo-Perú.

- $5^{\circ}$ Congreso Centroamericano de Fondos Viales organizado por el Fondo de Mantenimiento Vial (FOMAV) de Nicaragua, del 4 al 6 de mayo del 2011. Managua-Nicaragua.

- II Encuentro sobre Didáctica de la Estadística, la Probabilidad y el Análisis de Datos II EDEPA organizado por la Escuela de Matemáticas del Instituto Tecnológico de Costa Rica, del 28 al 29 de noviembre del 2011. CartagoCosta Rica

También se han dado cursos de capacitación, dirigida a profesionales en el diseño de canales y estudios hidrológicos:

- Colegio de Ingenieros Civiles 17, 24 setiembre, 01, 08 octubre del 2005, organizado por el Colegio de Ingenieros Electricistas, mecánicos e Industriales, San José, Costa Rica.

- Universidad Nacional del Altiplano y El Colegio de Ingenieros del Perú CD Puno, 11 al 13 de Setiembre del 2006, organizado por la Facultad de Ingeniería Agrícola. Puno-Perú.

- Plan Meriss Inka, del 05 al 08 de setiembre del 2007, organizado y patrocinado por Nicoll Perú S.A. CuscoPerú.

- Universidad Santo Tomás, del 01 al 02 de diciembre del 2011, Bogotá - Colombia.

Sirve como material didáctico, a través de la Ayuda que tiene el software y de su Manual del Usuario, y como herramienta de cálculo para los estudiantes de la Escuela de Ingeniería Agrícola, en los cursos de Estadística Aplica e Hidrología.

Es una tecnología computacional de cálculo que se pone a disposición de los Ingenieros Agrícolas, Civiles, Agrónomos, hidrólogos y demás profesionales relacionados con los estudios hidrológicos, de los diferentes países.

\section{Bibliografía}

[1] Haan, Ch. Statistical methods in hydrology. State University Press. 1977.

[2] Hosking, Jonathan. L-moments: Analysis and estimation of distributions using linear combinations of order statistics. Journal of the Royal Statistical Society B52: 105-124. 1990.

[3] Hosking, Jonathan. Some theoretical results concerning L-moments. Research Report, IBM Research Division. 1996.

[4] Hosking, Jonathan. Fortran routines for use with the meted of L-moments. Research Report, IBM Research Division. 2000.

[5] Kite, G.W. Frequency and risk analyses in hydrology. Water Resources Publications, Michigan. 1977.

[6] Maidment, David. Handbook of hydrology. Editorial McGraw-Hill. 1993. 
[7] Salas, José. Computer workshop in statistical hydrology. Hydrology and Water Resources Program, Colorado State University, Fort Collins. 1978.

[8] Villón, Máximo. Diseño de capacidad de embalses por el método experimental - teoría del rango. Tesis para optar por el grado de Magíster Scientiae en Ingeniería de Recursos de Agua y Tierra. Universidad Nacional Agraria "La Molina". 1983.

[9] Villón, Máximo. Hidrología. Editorial Tecnológica de Costa Rica. Instituto Tecnológico de Costa Rica. 2004.

[10] Villón, Máximo. Hidrología estadística. Editorial Tecnológica de Costa Rica. Instituto Tecnológico de Costa Rica. 2006. 